Document downloaded from:

http://hdl.handle.net/10251/160908

This paper must be cited as:

Alarcon, D.; Hueso, JL.; Martínez Molada, E. (2020). An alternative analysis for the local convergence of iterative methods for multiple roots including when the multiplicity is unknown. International Journal of Computer Mathematics. 97(1-2):312-329. https://doi.org/10.1080/00207160.2019.1589460

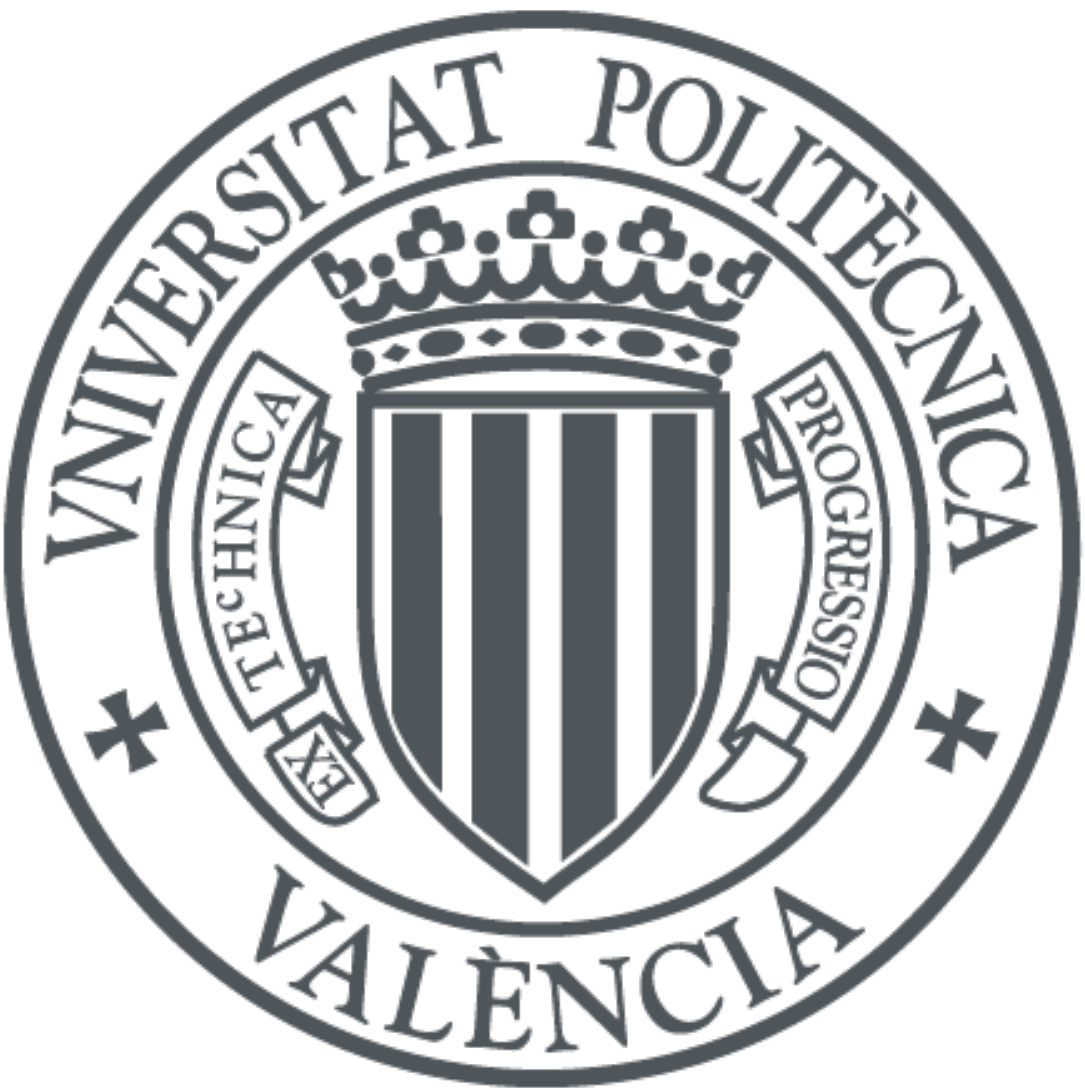

The final publication is available at

https://doi.org/10.1080/00207160.2019.1589460

Copyright Taylor \& Francis

Additional Information 


\title{
An alternative analysis for the local convergence of iterative methods for multiple roots including when the multiplicity is unknown
}

\author{
Diego Alarcón ${ }^{\mathrm{a}}$, Jose L. Hueso ${ }^{\mathrm{b}}$ and Eulalia Martínez ${ }^{\mathrm{b}}$ \\ a Departamento de Matemática Aplicada. Universitat Politècnica de València, Spain; \\ ${ }^{\mathrm{b}}$ Instituto Universitario de Matemática Multidisciplinar. Universitat Politècnica de València, \\ Spain
}

\section{ARTICLE HISTORY}

Compiled March 13, 2019

\begin{abstract}
In this paper we propose an alternative for the study of local convergence radius and the uniqueness radius for some third order methods for multiple roots whose multiplicity is known. The main goal is to provide an alternative that avoids the use of sophisticated properties of divided differences that are used in already published papers about local convergence for multiple roots. We defined the local study by using a technique taking into consideration a bounding condition for the $(m+i) t h$ derivative of the function $f(x)$ with $i=1,2$. In the case that the method uses first and second derivative in its iterative expression and $i=1$ in case the method only uses first derivative.

Furthermore we implement a numerical analysis in the following sense. Since the radius of local convergence for high-order methods decreases with the order, we must take into account the analysis of ITS behavior when we introduce a new iterative method. Finally, we have used these iterative methods for multiple roots for the case where the multiplicity $m$ is unknown, so we estimate this factor by different strategies comparing the behavior of the corresponding estimations and how this fact affect to the original method.
\end{abstract}

\section{KEYWORDS}

Nonlinear equations; Iterative methods; Multiple roots; Convergence Ball; Local convergence.

\section{Introduction}

In the last years some of the studies concerning iterative methods for approximating roots of nonlinear equations have focused on multiple roots. That is, to find a multiple zero $\gamma$ of multiplicity $m$ of a nonlinear equation $f(x)=0, f: D \subseteq: \mathbb{R} \longrightarrow: \mathbb{R}$, for this particular case some special aspects must be taken into account. This happens in the Van der Waals equation of state, in the compression of band-limited signals and the multipactor effect in electronic devices among other phenomenons. In this sense different iterative methods for this particular case have been recently published see [1]-[6] and the references therein.

For an iterative method, we define $r$ the radius of the local convergence ball if the sequence $x_{n}$ generated by this iterative method, starting from any initial point in 
the open ball $B(\gamma, r)$ converges to $\gamma$ and remains in the ball. In these studies it is interesting to obtain the largest possible value of $r$, but obviously, this depends on the conditions that the nonlinear function verifies.

Specially interesting from a mathematical point of view is paper [1] where a complete local convergence study has been performed, obtaining the convergence radius of the well known modified Newtons method for multiple zeros,

$$
x_{n+1}=x_{n}-m \frac{f\left(x_{n}\right)}{f^{\prime}\left(x_{n}\right)}
$$

when the involved function satisfies Hölder continuity conditions, that is, $\forall x, y \in D$, $p \in] 0,1]$ and $K_{0}, K_{m}$ positive real numbers.

$$
\begin{aligned}
& \left|f^{(m)}\left(x^{*}\right)^{-1}\left(f^{(m+1)}(x)-f^{(m+1)}(y)\right)\right| \leq K_{0}|x-y|^{p}, \\
& \left|f^{(m)}\left(x^{*}\right)^{-1} f^{(m+1)}(x)\right| \leq K_{m} .
\end{aligned}
$$

For this purpose, different results involving divided differences have been used. The same sophisticated properties have been used for obtaining the local convergence study of the third order Halley's method, [3], given by:

$$
x_{n+1}=x_{n}-\frac{f\left(x_{n}\right)}{\frac{m+1}{2 m} f^{\prime}\left(x_{n}\right)-\frac{f^{\prime}\left(x_{n}\right) f^{\prime \prime}\left(x_{n}\right)}{2 f^{\prime}\left(x_{n}\right)}},
$$

and Osada's method, [13], whose function iteration is:

$$
x_{n+1}=x_{n}-\frac{1}{2} m(m+1) \frac{f\left(x_{n}\right)}{f^{\prime}\left(x_{n}\right)}+\frac{1}{2}(m-1)^{2} \frac{f^{\prime}\left(x_{n}\right)}{f^{\prime \prime}\left(x_{n}\right)} .
$$

In this paper we propose an alternative to obtain this local convergence radius for iterative methods for nonlinear equations with roots with multiplicity $m$ showing that we can obtain similar results in a much more natural way. The bound conditions (6) and $(7)$ used for this work for a function $f$ that defines the nonlinear problem are shown below.

$$
\left|f^{(m)}(\gamma)^{-1} f^{(m+1)}(x)\right| \leq k, \quad \forall x \in D, \quad k>0 .
$$

For the method that uses second derivative we need to add another assumption as follows:

$$
\left|f^{(m)}(\gamma)^{-1} f^{(m+2)}(x)\right| \leq p, \quad \forall x \in D, \quad p>0 .
$$

First of all we develop the whole study for the third order iterative method due to Osada whose iterative expression is given by (5), we obtained similar results for local radius that the ones obtained in precedent studies cited before. 
An analogous procedure can be applied in order to obtain the radius of other iterative methods. Specifically we have also studied the third order method introduced by Dong in [6], whose expression is given by:

$$
\begin{aligned}
& y_{n}=x_{n}-\sqrt{m} \frac{f\left(x_{n}\right)}{f^{\prime}\left(x_{n}\right)}, \\
& x_{n+1}=y_{n}-m\left(1-\frac{1}{\sqrt{m}}\right)^{(1-m)} \frac{f\left(y_{n}\right)}{f^{\prime}\left(x_{n}\right)},
\end{aligned}
$$

this method has two steps and for this reason some variations must be taken into account.

\section{Preliminaries}

In this study we use a simple form for studying the local convergence radius of some iterative method for approximating multiple roots, for that we require the Taylor expansion with integral form remainder, avoiding in this way the use of some intricate properties of divided differences operator that were used in previous works with this aim.

Lemma 2.1. If $\gamma$ is a multiple zero of multiplicity $m$ with $m>1$, of a nonlinear equation $f(x)=0$, where $f: D \rightarrow R$ is a sufficiently differentiable function in a open interval $D$, then the function $f(x)$ can be expressed as

$$
f(x)=(x-\gamma)^{m} h(x), \quad h(\gamma) \neq 0,
$$

where

$$
h(x)=\frac{f^{(m)}(\gamma)}{m !}+\frac{1}{(m-1) !} \int_{0}^{1}\left[f^{(m)}(\gamma+\theta(x-\gamma))-f^{(m)}(\gamma)\right](1-\theta)^{m-1} d \theta,
$$

and it verifies

$$
h(\gamma)=\frac{f^{(m)}(\gamma)}{m !}
$$

and their derivatives for $i=1,2$ are

$$
h^{(i)}(x)=\frac{1}{(m-1) !} \int_{0}^{1} f^{(m+1)}(\gamma+\theta(x-\gamma)) \theta^{i}(1-\theta)^{m-1} d \theta .
$$

Proof: It is well known that if the nonlinear equation $f(x)=0$ has $\gamma$ a zero of multiplicity $m$ and $m>1$, then, it verifies $f^{(j)}(\gamma)=0, \quad j=0,1, \cdots, m-1$, and $f^{(m)}(\gamma) \neq 0$, and function $f$ can be expressed as:

$$
f(x)=(x-\gamma)^{m} g(x), \quad g(\gamma) \neq 0 .
$$

Approximating the function $f(x)$ by the Taylor expansion with integral form remainder 
around $\gamma,[8]$ we obtain

$$
\begin{aligned}
f(x) & =\frac{f^{(m)}(\gamma)}{m !}(x-\gamma)^{m}+\int_{\gamma}^{x} \frac{f^{(m+1)}(t)}{m !}(x-t)^{m} d t \\
& =\frac{f^{(m)}(\gamma)}{m !}(x-\gamma)^{m}+\frac{1}{(m-1) !} \int_{\gamma}^{x}\left[f^{(m)}(t)-f^{(m)}(\gamma)\right](x-t)^{m-1} d t \\
& =\frac{f^{(m)}(\gamma)}{m !}(x-\gamma)^{m}+\frac{1}{(m-1) !} \int_{0}^{1}\left[f^{(m)}(\gamma+\theta(x-\gamma))-f^{(m)}(\gamma)\right](x-\gamma)^{m}(1-\theta)^{m-1} d \theta \\
& =\left[\frac{f^{(m)}(\gamma)}{m !}+\frac{1}{(m-1) !} \int_{0}^{1}\left[f^{(m)}(\gamma+\theta(x-\gamma))-f^{(m)}(\gamma)\right](1-\theta)^{m-1} d \theta\right](x-\gamma)^{m},
\end{aligned}
$$

where one can check second equality by writing the last integral as $\int_{\gamma}^{x} u d v$ with $u=f^{(n)}(t)-f^{n}(\gamma)$ and $d v=(x-t)^{n-1} d t$.

Then, by comparing the zero $\gamma$ of multiplicity $m$ of function (13) with the one of (9), we see that they have the same structure, therefore we can deduce (10). Expressions, (11) and (12) follows obviously.

This property suggests that $f$ satisfies the boundary conditions established in (6) and (7), which allows establishing the following bounds that we will use in our study.

Lemma 2.2. Let $f(x)$ be a function satisfying conditions (6) and (7) for all $x_{0} \in$ ]$\gamma-r_{0}, \gamma+r_{0}\left[=I_{0}\right.$ where $r_{0}=\frac{m+1}{k}$ and $e_{0}=x_{0}-\gamma$. Then, function $h(x)$ defined by (10) verifies the following bounds:

$$
\begin{aligned}
& \left(B_{1}\right) \quad\left|h(\gamma)^{-1} h\left(x_{0}\right)\right| \leq \frac{m+1+k\left|e_{0}\right|}{m+1} \\
& \left(B_{2}\right) \quad\left|h(\gamma)^{-1} h^{\prime}\left(x_{0}\right)\right| \leq \frac{k}{m+1} \\
& \left(B_{3}\right) \quad\left|h\left(x_{0}\right)^{-1} h(\gamma)\right| \leq \frac{m+1}{m+1-k\left|e_{0}\right|} \\
& \left(B_{4}\right) \quad\left|h\left(x_{0}\right)^{-1} h^{\prime}\left(x_{0}\right)\right| \leq \frac{k}{m+1-k\left|e_{0}\right|} \\
& \left(B_{5}\right) \quad\left|h\left(x_{0}\right)^{-1} h^{\prime \prime}\left(x_{0}\right)\right| \leq \frac{2 p}{(m+2)\left(m+1-k\left|e_{0}\right|\right)} .
\end{aligned}
$$

Proof: Notice that by Lemma 2.1 it we can be deduced that $h(\gamma)^{-1} \neq 0$ so by using assumptions above defined, (6) and (7), and applying the Mean Value Theorem we have $\left(B_{1}\right)$,

$$
\begin{aligned}
\left|h(\gamma)^{-1} h\left(x_{0}\right)\right| & =\left|1+m \int_{0}^{1} f^{(m)}(\gamma)^{-1}\left[f^{(m)}\left(\gamma+\theta\left(x_{0}-\gamma\right)\right)-f^{(m)}(\gamma)\right](1-\theta)^{m-1} d \theta\right| \\
& \leq\left|1+m \int_{0}^{1} f^{(m)}(\gamma)^{-1} f^{(m+1)}(\xi) \theta\right| e_{0}\left|(1-\theta)^{m-1} d \theta\right| \leq \frac{m+1+k\left|e_{0}\right|}{m+1},
\end{aligned}
$$

where in the last inequality we have used that $\int_{0}^{1} \theta(1-\theta)^{m-1} d \theta=\frac{1}{m(m+1)}$. 
Similar reasoning allow us to get $\left(B_{2}\right)$

$$
\left|h(\gamma)^{-1} h^{\prime}\left(x_{0}\right)\right|=\left|m ! f^{(m)}(\gamma)^{-1} \frac{1}{(m-1) !} \int_{0}^{1} f^{(m+1)}\left(\gamma+\theta\left(x_{0}-\gamma\right)\right) \theta(1-\theta)^{m-1} d \theta\right| \leq \frac{k}{m+1} .
$$

Now for getting $\left(B_{3}\right)$ we use the Mean Value Theorem having $\xi$ between $\gamma$ and $x_{0}$ such as:

$$
\left|1-h(\gamma)^{-1} h\left(x_{0}\right)\right|=\left|h(\gamma)^{-1}\left(h(\gamma)-h\left(x_{0}\right)\right)\right|=\left|h(\gamma)^{-1} h^{\prime}(\xi)\right| e_{0}|| \leq \frac{k\left|e_{0}\right|}{m+1}<1,
$$

so, we can apply Banach Lemma to establish the existence of $h\left(x_{0}\right)^{-1}$ and moreover it is verified that:

$$
\left|h\left(x_{0}\right)^{-1} h(\gamma)\right| \leq \frac{m+1}{m+1-k\left|e_{0}\right|}
$$

Following bounds $\left(B_{4}\right)$ and $\left(B_{5}\right)$ can be achieved by using previous ones.

\section{Local convergence results}

\subsection{Local convergence for a one step iterative method}

In this section in order to obtain the local convergence radius for the third order method defined by (5) we analyze it by taking an initial guess $\left.x_{0} \in\right] \gamma-r, \gamma+r\left[=I_{r}\right.$ where $r<r_{0}$. For this we obtain the error equation of this method for obtaining the iteration $x_{1}$ generating some more restrictions that will determine the final value for $r$. In the process we use bounds obtained in Lemma 2.2 and after that we will follow an induction procedure to complete the study.

So, the first iteration can be written as

$$
x_{1}=x_{0}-\frac{1}{2} m(m+1) \frac{f\left(x_{0}\right)}{f^{\prime}\left(x_{0}\right)}+\frac{1}{2}(m-1)^{2} \frac{f^{\prime}\left(x_{0}\right)}{f^{\prime \prime}\left(x_{0}\right)} .
$$

Then, from Lemma 2.1 and being $\gamma$ a zero of multiplicity $m$ of equation $f(x)=0$ and $e_{0}=x_{0}-\gamma$ we obtain,

$$
\begin{aligned}
f\left(x_{0}\right) & =h\left(x_{0}\right) e_{0}^{m}, \\
f^{\prime}\left(x_{0}\right) & =h^{\prime}\left(x_{0}\right) e_{0}^{m}+m h\left(x_{0}\right) e_{0}^{m-1} \\
f^{\prime \prime}\left(x_{0}\right) & =h^{\prime \prime}\left(x_{0}\right) e_{0}^{m}+2 m h^{\prime}\left(x_{0}\right) e_{0}^{m-1}+m(m-1) h\left(x_{0}\right) e_{0}^{m-2} .
\end{aligned}
$$

So, by subtracting $\gamma$ in both sides of (2) and using these expressions we have:

$$
\begin{aligned}
e_{1} & =e_{0}-\frac{1}{2} m(m+1) \frac{h\left(x_{0}\right) e_{0}^{m}}{h^{\prime}\left(x_{0}\right) e_{0}^{m}+m h\left(x_{0}\right) e_{0}^{m-1}} \\
& +\frac{1}{2}(m-1)^{2} \frac{h^{\prime}\left(x_{0}\right) e_{0}^{m}+m h\left(x_{0}\right) e_{0}^{m-1}}{h^{\prime \prime}\left(x_{0}\right) e_{0}^{m}+2 m h^{\prime}\left(x_{0}\right) e_{0}^{m-1}+m(m-1) h\left(x_{0}\right) e_{0}^{m-2}},
\end{aligned}
$$


then we write the error equation as:

$$
e_{1}=\frac{A}{B} e_{0}
$$

where

$$
A=2 h^{\prime}\left(x_{0}\right) h^{\prime \prime}\left(x_{0}\right) e_{0}^{3}+(m+1)^{2} h^{\prime}\left(x_{0}\right)^{2} e_{0}^{2}+m(1-m) h\left(x_{0}\right) h^{\prime \prime}\left(x_{0}\right) e_{0}^{2},
$$

and

$$
\begin{aligned}
B & =2 h^{\prime}\left(x_{0}\right) h^{\prime \prime}\left(x_{0}\right) e_{0}^{3}+4 m h^{\prime}\left(x_{0}\right)^{2} e_{0}^{2}+2 m(3 m-1) h\left(x_{0}\right) h^{\prime}\left(x_{0}\right) e_{0} \\
& +2 m h\left(x_{0}\right) h^{\prime \prime}\left(x_{0}\right) e_{0}^{2}+2 m^{2}(m-1) h\left(x_{0}\right)^{2}
\end{aligned}
$$

Now, by dividing both terms by the expression $2 m^{2}(m-1) h(\gamma) h\left(x_{0}\right)$ denoting new terms $\hat{A}$ and $\hat{B}$, we have:

$$
e_{1}=\frac{\hat{A}}{\hat{B}} e_{0}
$$

Then, in order to bound the quotient $\left|e_{1}\right|$, we bound upperly the numerator then,

$$
\begin{aligned}
|\hat{A}| & =\mid \frac{h(\gamma)^{-1} h^{\prime}\left(x_{0}\right) h\left(x_{0}\right)^{-1} h^{\prime \prime}\left(x_{0}\right) e_{0}^{3}}{m^{2}(m+1)}+\frac{(m+1)^{2} h(\gamma)^{-1} h^{\prime}\left(x_{0}\right) h\left(x_{0}\right)^{-1} h^{\prime}\left(x_{0}\right) e_{0}^{2}}{2 m^{2}(m+1)} \\
& -\frac{h(\gamma)^{-1} h^{\prime \prime}\left(x_{0}\right) h\left(x_{0}\right)^{-1} h\left(x_{0}\right) e_{0}^{2}}{2 m} \mid
\end{aligned}
$$

by using bounds obtained in Lemma 2.2 we get

$$
|\hat{A}| \leq \frac{2 k p\left|e_{0}\right|^{3}}{m^{2}\left(m^{2}-1\right)(m+2)\left(m+1-k\left|e_{0}\right|\right)}+\frac{(m+1) k^{2}\left|e_{0}\right|^{2}}{2 m^{2}(m-1)\left(m+1-k\left|e_{0}\right|\right)}+\frac{p\left|e_{0}\right|^{2}}{m(m+1)(m+2)}
$$

So, we have $|\hat{A}| \leq \varphi\left(\left|e_{0}\right|\right)$ with $\varphi:\left[0, r_{0}[\quad \rightarrow \quad \mathbb{R}\right.$, defined as:

$$
\varphi(t)=\frac{4 k p t^{3}+\left[(m+1)^{2}(m+2) k^{2}+2 m(m-1)(m+1-k t)\right] t^{2}}{2 m^{2}\left(m^{2}-1\right)(m+2)(m+1-k t)}
$$

and one can check that $\varphi$ is a increasing function in $\left[0, r_{0}[\right.$ for being the quotient of two positive functions, an increasing numerator and a decreasing denominator.

After that, we study the term $\hat{B}$ of (17) as follows:

$$
\begin{aligned}
|\hat{B}| & =\mid \frac{h(\gamma)^{-1} h^{\prime}\left(x_{0}\right) h\left(x_{0}\right)^{-1} h^{\prime \prime}\left(x_{0}\right) e_{0}^{3}}{m^{2}(m-1)}+\frac{2 h(\gamma)^{-1} h^{\prime}\left(x_{0}\right) h\left(x_{0}\right)^{-1} h^{\prime}\left(x_{0}\right) e_{0}^{2}}{m(m-1)} \\
& +\frac{h(\gamma)^{-1} h^{\prime \prime}\left(x_{0}\right) e_{0}^{2}}{m(m-1)}+\frac{(3 m-1) h(\gamma)^{-1} h^{\prime}\left(x_{0}\right) e_{0}}{m(m-1)}+h(\gamma)^{-1} h\left(x_{0}\right) \mid .
\end{aligned}
$$


In order to apply Banach Lemma we have:

$$
\begin{aligned}
|I-\hat{B}| & \leq \mid h(\gamma)^{-1} h(\gamma)-h(\gamma)^{-1} h\left(x_{0}\right)-\frac{h(\gamma)^{-1} h^{\prime}\left(x_{0}\right) h\left(x_{0}\right)^{-1} h^{\prime \prime}\left(x_{0}\right) e_{0}^{3}}{m^{2}(m-1)}-\frac{2 h(\gamma)^{-1} h^{\prime}\left(x_{0}\right) h\left(x_{0}\right)^{-1} h^{\prime}\left(x_{0}\right) e_{0}^{2}}{m(m-1)} \\
& -\frac{h(\gamma)^{-1} h^{\prime \prime}\left(x_{0}\right) e_{0}^{2}}{m(m-1)}-\frac{(3 m-1) h(\gamma)^{-1} h^{\prime}\left(x_{0}\right) e_{0}}{m(m-1)} \mid .
\end{aligned}
$$

So by using the Mean Value Theorem for the first term and bounds obtained in Lemma 2.2 we get

$$
\begin{aligned}
|I-\hat{B}| & \leq \frac{k\left|e_{0}\right|}{m+1}+\frac{2 k p\left|e_{0}\right|^{3}}{m^{2}\left(m^{2}-1\right)(m+2)\left(m+1-k\left|e_{0}\right|\right)}+\frac{2 k^{2}\left|e_{0}\right|^{2}}{m\left(m^{2}-1\right)\left(m+1-k\left|e_{0}\right|\right)} \\
& +\frac{2 p\left|e_{0}\right|^{2}}{m\left(m^{2}-1\right)(m+2)}+\frac{(3 m-1) k\left|e_{0}\right|}{m\left(m^{2}-1\right)} \leq g_{1}\left(\left|e_{0}\right|\right)
\end{aligned}
$$

where $g_{1}$ is defined as:

$$
\begin{aligned}
g_{1}(t) & =\frac{2 k p}{m^{2}\left(m^{2}-1\right)(m+2)(m+1-k t)} t^{3}+\frac{2\left[(m+2) k^{2}+(m+1-k t) p\right]}{m\left(m^{2}-1\right)(m+2)(m+1-k t)} t^{2} \\
& +\frac{\left(m^{2}+2 m-1\right) k}{m\left(m^{2}-1\right)} t .
\end{aligned}
$$

Notice that, $g_{1}$ is an increasing function in $] 0, r_{0}[$, for being the quotient of two positive functions, increasing the numerator and decreasing the denominator, so, we take $h_{1}(t)=g_{1}(t)-1$, verifying, $h_{1}(0)=-1$ and $h_{1}\left(r_{0}^{-}\right) \rightarrow+\infty$. Then $h_{1}$ has a unique a root $r_{1}$ between these values, where $\left.r_{1} \in\right] 0, r_{0}\left[/ 0 \leq g_{1}(t)<1, \forall t \in\right] 0, r_{1}[$, therefore by applying Banach's Lemma it follows:

$$
\left|\hat{B}^{-1}\right| \leq \frac{1}{1-g_{1}\left(\left|e_{0}\right|\right)}
$$

Then, turning to (17) we get

$$
\left|e_{1}\right| \leq\left|\hat{B}^{-1} \hat{A}\right|\left|e_{0}\right| \leq \frac{\varphi\left(\left|e_{0}\right|\right)}{1-g_{1}\left(\left|e_{0}\right|\right)}\left|e_{0}\right|=g_{2}\left(\left|e_{0}\right|\right)\left|e_{0}\right|
$$

where

$$
g_{2}(t)=\frac{\varphi(t)}{1-g_{1}(t)}
$$

is an increasing function in $] 0, r_{1}\left[\right.$. So if we take $h_{2}(t)=g_{2}(t)-1$, it is verified $h_{2}(0)=-1<0$ and $h_{2}\left(r_{1}^{-}\right) \rightarrow+\infty$. Therefore $\left.\exists r_{2} \in\right] 0, r_{1}\left[/ 0 \leq g_{2}(t)<1 \forall t \in\right] 0, r_{2}[$.

Now, as we have obtained the sequence $r_{2}<r_{1}<r_{0}$ we take $r=r_{2}$, concluding that: 


$$
\left.e_{1}=\left|x_{1}-\gamma\right| \leq g_{2}\left(\left|x_{0}-\gamma\right|\right)\left|x_{0}-\gamma\right|<\left|x_{0}-\gamma\right|=e_{0}, \quad \forall x_{0} \in\right] \gamma-r, \gamma+r[
$$

That is, $\left.x_{1} \in\right] \gamma-r, \gamma+r\left[=I\right.$. The same process holds starting from $x_{1}$ and getting $x_{2}$ and then it is obtained that for all $k>0$ it follows:

$$
e_{k+1}=\left|x_{k+1}-\gamma\right| \leq g_{2}\left(\left|x_{k}-\gamma\right|\right)\left|x_{k}-\gamma\right|<\left|x_{k}-\gamma\right|
$$

So we have obtained that:

$$
\left|e_{k+1}\right| \leq g_{2}(r)\left|e_{k}\right| \leq\left(g_{2}(r)\right)^{2}\left|e_{k-1}\right| \leq \cdots \leq\left(g_{2}(r)\right)^{k+1}\left|e_{0}\right| .
$$

Then, we deduce that $\left.x_{k+1} \in\right] \gamma-r, \gamma+r[=I$ and by taking limits in the last expression and using that $\lim _{k \rightarrow+\infty}\left(g_{2}(r)\right)^{k+1}=0$, we get that $\lim _{k \rightarrow+\infty} x_{k}=\gamma$ so we have proved the following

Theorem 3.1. Under the conditions of Lemma 2.2, let $r_{0}=\frac{m+1}{k}$ and $\left.r_{i}, i=1,2\right\}$ be the unique positive root of equation $h_{i}(t)=g_{i}(t)-1, i=1,2$, with functions $g_{i}$ defined below. Then, by taking $r=r_{2}$, for any initial guess $\left.x_{0} \in\right] \gamma-r, \gamma+r[$ the sequence $\left\{x_{k}\right\}$ obtained by the iterative method defined in (5) is well defined verifying that $\left.x_{k+1} \in\right] \gamma-r, \gamma+r[$ for all $k \geq 0$ and converges at a rate of order at least 3 to the unique solution $\left.x^{*} \in\right] \gamma-r_{0}, \gamma+r_{0}[$. Moreover, the following error bound holds for all $n \geq 0$

$$
\left|e_{k+1}\right| \leq \frac{\left|e_{k}\right|^{3}}{r_{2}^{3}}
$$

Proof: The first part of the proof follows from results of previous section. In order to obtain the rate of convergence turning to (18) and by using definitions of function $\varphi$, we have:

$$
e_{1} \leq\left|e_{0}\right| \frac{\frac{4 k p\left|e_{0}\right|^{3}+\left[(m+1)^{2}(m+2) k^{2}+2 m(m-1)\left(m+1-k\left|e_{0}\right|\right)\right]\left|e_{0}\right|^{2}}{2 m^{2}\left(m^{2}-1\right)(m+2)\left(m+1-k\left|e_{0}\right|\right)}}{1-g_{1}\left(\left|e_{0}\right|\right)} .
$$

Now, we multiply and divide by $\left|e_{0}\right|^{2}$

$$
e_{1} \leq\left|e_{0}\right|^{3} \frac{\frac{4 k p\left|e_{0}\right|+\left[(m+1)^{2}(m+2) k^{2}+2 m(m-1)\left(m+1-k\left|e_{0}\right|\right)\right]}{2 m^{2}\left(m^{2}-1\right)(m+2)\left(m+1-k\left|e_{0}\right|\right)}}{1-g_{1}\left(\left|e_{0}\right|\right)}
$$

observe that the function that we obtain in the numerator is increasing in $I_{0}$, so we 
have by the definition of $r_{2}$ that:

$$
\begin{aligned}
e_{1} \leq & \frac{\left|e_{0}\right|^{3}}{r_{2}^{2}} \frac{\left(\frac{4 k p r_{2}+\left[(m+1)^{2}(m+2) k^{2}+2 m(m-1)\left(m+1-k r_{2}\right)\right]}{2 m^{2}\left(m^{2}-1\right)(m+2)\left(m+1-k r_{2}\right)}\right) r_{2}^{2}}{1-g_{1}\left(r_{2}\right)} \\
& =\frac{\left|e_{0}\right|^{3}}{r_{2}^{2}} g_{2}\left(r_{2}\right)=\frac{\left|e_{0}\right|^{3}}{r_{2}^{2}} .
\end{aligned}
$$

Then, by an induction procedure we have $\left|e_{k+1}\right| \leq \frac{\left|e_{k}\right|^{3}}{r_{2}^{2}}$, that is, sequence $x_{k}$ converges to $\gamma$ with order at least 3 .

To show the uniqueness, we assume that there exists a second solution $\left.y^{*} \in\right] \gamma-$ $r_{0}, \gamma+r_{0}[$, by (9) we have

$$
f\left(y^{*}\right)=h\left(y^{*}\right)\left(y^{*}-\gamma\right)^{m} .
$$

By using the Mean Value Theorem and Lemma 2.2 we have:

$$
\left|1-h(\gamma)^{-1} h\left(y^{*}\right)\right|=\left|h(\gamma)^{-1}\left(h(\gamma)-h\left(y^{*}\right)\right)\right|=\left|h(\gamma)^{-1} h^{\prime}(\xi)\right| \gamma-y^{*}|| \leq \frac{k\left|\gamma-y^{*}\right|}{m+1}<1,
$$

we deduce that $h\left(y^{*}\right) \neq 0$ and then by $(20)$ we have that $y^{*}=\gamma$.

\subsection{Local convergence for a two steps iterative method}

Now in order to obtain the local convergence radius for the third order method defined by (8) we analyze their iterates by taking an initial guess $\left.x_{0} \in\right] \gamma-r, \gamma+r[$, where $r<r_{0}$. This value will be determinaded once the error equation of this method is obtained and analyzed. In the process we use bounds obtained in Lemma 2.2 and after that we will follow an induction procedure to complete the study in an analogous was as it has been done with the previous method.

So, by taking an initial guess $\left.x_{0} \in\right] \gamma-r, \gamma+r\left[\right.$, where $r<r_{0}$ using expressions obtained in (15) from the the first step of (8) we can write:

$$
\hat{e}_{0}=x_{0}-\gamma-m^{\frac{1}{2}} \frac{f\left(x_{0}\right)}{f^{\prime}\left(x_{0}\right)}=\frac{h^{\prime}\left(x_{0}\right) e_{0}+m^{\frac{1}{2}}\left(m^{\frac{1}{2}}-1\right) h\left(x_{0}\right)}{h^{\prime}\left(x_{0}\right) e_{0}+m h\left(x_{0}\right)} e_{0}
$$

Multiplying both terms by the expression $m^{-1} h(\gamma)^{-1}$ we have

$$
\hat{e}_{0}=\frac{\hat{A}_{0}}{\hat{B}_{0}} e_{0}
$$

with

$$
\begin{aligned}
& \hat{A}_{0}=m^{-1} h(\gamma)^{-1}\left(h^{\prime}\left(x_{0}\right) e_{0}+m^{\frac{1}{2}}\left(m^{\frac{1}{2}}-1\right) h\left(x_{0}\right)\right), \\
& \hat{B}_{0}=m^{-1} h(\gamma)^{-1}\left(h^{\prime}\left(x_{0}\right) e_{0}+m h\left(x_{0}\right)\right) .
\end{aligned}
$$


Now, applying bounds obtained in Lemma 2.2 and taking into account that $m^{\frac{1}{2}}\left(m^{\frac{1}{2}}-1\right)>0$ it follows:

$$
\left|\hat{A}_{0}\right| \leq \frac{k\left|e_{0}\right|+m^{\frac{1}{2}}\left(m^{\frac{1}{2}}-1\right)\left(m+1+k\left|e_{0}\right|\right)}{m(m+1)} .
$$

In order to use Banach's Lemma we calculate

$$
\left|I-\hat{B}_{0}\right|=\left|h(\gamma)^{-1}\left(h(\gamma)-h\left(x_{0}\right)\right)-m^{-1} h(\gamma)^{-1} h^{\prime}\left(x_{0}\right) e_{0}\right| \leq \frac{k\left|e_{0}\right|}{m},
$$

then, we need $\frac{k\left|e_{0}\right|}{m}<1$, so we have to take now $\left|e_{0}\right|<\frac{m}{k}=r_{1}$ and being this value minor than $r_{0}=\frac{m+1}{k}$ defined in Lemma 2.2 we are in conditions of applying the bounds established there. So we have:

$$
\left|\hat{B}_{0}^{-1}\right|=\frac{m}{m-k\left|e_{0}\right|},
$$

and so turning to $(21)$ one has:

$$
\left|\hat{e}_{0}\right| \leq \frac{k\left|e_{0}\right|+m^{\frac{1}{2}}\left(m^{\frac{1}{2}}-1\right)\left(m+1+k\left|e_{0}\right|\right)}{(m+1)\left(m-k\left|e_{0}\right|\right)}\left|e_{0}\right|=g_{1}\left(\left|e_{0}\right|\right)\left|e_{0}\right|,
$$

where

$$
g_{1}(t)=\frac{k t+m^{\frac{1}{2}}\left(m^{\frac{1}{2}}-1\right)(m+1+k t)}{(m+1)(m-k t)}
$$

is an increasing function in $] 0, r_{1}\left[\right.$. Then by taking $h_{1}(t)=g_{1}(t)-1$, it is verified that $h_{1}(0)=-m^{-\frac{1}{2}}$ and $h_{1}\left(r_{1}^{-}\right) \rightarrow+\infty$. Therefore $h_{1}$ has a unique root in $] 0, r_{1}[$ let it be $r_{2}$, then we have that $\left.0<g_{1}(t)<1 \forall t \in\right] 0, r_{2}$ [ and so:

$$
\left|y_{0}-\gamma\right| \leq g_{1}\left(\left|x_{0}-\gamma\right|\right)\left|x_{0}-\gamma\right|<\left|x_{0}-\gamma\right| .
$$

Now, we proceed to analyze the second step of (8) by similar expressions to the ones in (15) but now with $\hat{e}_{0}=y_{0}-\gamma$ we have:

$$
\begin{aligned}
f\left(y_{0}\right) & =h\left(y_{0}\right) \hat{e}_{0}^{m}, \\
f^{\prime}\left(y_{0}\right) & =h^{\prime}\left(y_{0}\right) \hat{e}_{0}^{m}+m h\left(y_{0}\right) \hat{e}_{0}^{m-1},
\end{aligned}
$$

substituting these in

$$
x_{1}=y_{0}-m b \frac{f\left(y_{0}\right)}{f^{\prime}\left(x_{0}\right)},
$$


and dividing numerator and denominator by $e_{0}^{m-1}$ we get:

$$
e_{1}=x_{1}-\gamma=\hat{e}_{0}-\frac{m b h\left(y_{0}\right)\left(\frac{\hat{e}_{0}}{e_{0}}\right)}{h^{\prime}\left(x_{0}\right) e_{0}+m h\left(x_{0}\right)} e_{0}
$$

By doing some calculations and denoting $N=\frac{\hat{e}_{0}}{e_{0}}$ we write the error equation as:

$$
e_{1}=\frac{A_{1}}{B_{0}} e_{0}
$$

where $B_{0}$ has been used in (21) for first step and

$$
A_{1}=h^{\prime}\left(x_{0}\right) e_{0}+m^{\frac{1}{2}}\left(m^{\frac{1}{2}}-1\right) h\left(x_{0}\right)-m b h\left(y_{0}\right) N^{m},
$$

so, by dividing both terms by the expression $m h(\gamma)$ denoting new terms $\hat{A}_{1}$ and $\hat{B}_{0}$, we have:

$$
e_{1}=\frac{\hat{A}_{1}}{\hat{B}_{0}} e_{0}
$$

Now, we analyze $\left|e_{1}\right|$, for this, we first bound the numerator given by:

$$
\left|\hat{A}_{1}\right|=\left|m^{-1} h(\gamma)^{-1} h^{\prime}\left(x_{0}\right) e_{0}+m^{-\frac{1}{2}}\left(m^{\frac{1}{2}}-1\right) h(\gamma)^{-1} h\left(x_{0}\right)-b h(\gamma)^{-1} h\left(y_{0}\right) N^{m}\right|
$$

and using bounds obtained in Lemma 2.2 we get

$$
\left|\hat{A}_{1}\right| \leq \frac{k\left|e_{0}\right|}{m(m+1)}+\frac{\left(m^{\frac{1}{2}}-1\right)\left(m+1+k\left|e_{0}\right|\right)}{m^{\frac{1}{2}}(m+1)}+\frac{b\left(m+1+k g_{1}\left(\left|e_{0}\right|\right)\left|e_{0}\right|\right) g_{1}\left(\left|e_{0}\right|\right)^{m}}{m+1} .
$$

So, we have obtained $\left|\hat{A}_{1}\right| \leq \varphi\left(\left|e_{0}\right|\right)$ with $\varphi:\left[0, r_{1}[\quad \longrightarrow \quad \mathbb{R}\right.$, defined as:

$$
\varphi(t)=\frac{k t+m^{\frac{1}{2}}\left(m^{\frac{1}{2}}-1\right)(m+1+k t)+m b\left(m+1+k g_{1}(t) t\right) g_{1}(t)^{m}}{m(m+1)}
$$

and one can check that $\varphi$ is a increasing function because $g_{1}$ is so.

Then, turning to (25) and using (22) we get:

$$
\left|\hat{e}_{1}\right| \leq \frac{\varphi\left(\left|e_{0}\right|\right)}{(m+1)\left(m-k\left|e_{0}\right|\right)}
$$

that is,

$$
\begin{aligned}
\left|\hat{e}_{1}\right| & \leq \frac{k\left|e_{0}\right|+m^{\frac{1}{2}}\left(m^{\frac{1}{2}}-1\right)\left(m+1+k\left|e_{0}\right|\right)+m b\left(m+1+k g_{1}\left(\left|e_{0}\right|\right)\left|e_{0}\right|\right) g_{1}\left(\left|e_{0}\right|\right)^{m}}{(m+1)\left(m-k\left|e_{0}\right|\right)}\left|e_{0}\right| \\
& \leq g_{2}\left(\left|e_{0}\right|\right)\left|e_{0}\right|,
\end{aligned}
$$


where

$$
g_{2}(t)=\frac{k t+m^{\frac{1}{2}}\left(m^{\frac{1}{2}}-1\right)(m+1+k t)+m b\left(m+1+k g_{1}(t) t\right) g_{1}(t)^{m}}{(m+1)(m-k t)}
$$

and we take $h_{2}(t)=g_{2}(t)-1$, verifying, $h_{2}(0)=\frac{m^{\frac{1}{2}}-1}{m^{\frac{1}{2}}}+b\left(\frac{m^{\frac{1}{2}}-1}{m^{\frac{1}{2}}}\right)^{m}-1<0$ and $h_{2}\left(r_{1}^{-}\right) \rightarrow+\infty$. Then $h_{2}(t)$ has a unique root between these values, let it be $r_{3}$. Therefore $\left.\exists r_{3} \in\right] 0, r_{1}\left[/ 0 \leq g_{2}(t)<1 \forall t \in\right] 0, r_{3}[$.

Now, we take $r=\min \left\{r_{i}, i=0, \cdots, 3\right\}$ for concluding that:

$$
\begin{aligned}
& \left|y_{0}-\gamma\right| \leq g_{1}\left(\left|x_{0}-\gamma\right|\right)\left|x_{0}-\gamma\right|<\left|x_{0}-\gamma\right|, \\
& \left|x_{1}-\gamma\right| \leq g_{2}\left(\left|x_{0}-\gamma\right|\right)\left|x_{0}-\gamma\right|<\left|x_{0}-\gamma\right|,
\end{aligned}
$$

That is, $\left.y_{0}, x_{1} \in\right] \gamma-r, \gamma+r\left[=I\right.$. The same process holds starting from $x_{1}$ and getting $y_{1}, x_{2}$ and then by and inductive procedure it is obtained that for all $k>0$ it follows:

$$
\begin{aligned}
\left|y_{k}-\gamma\right| & \leq\left|g_{1}\left(\left|x_{k}-\gamma\right|\right)\right| x_{k}-\gamma|<| x_{k}-\gamma \mid, \\
\left|x_{k+1}-\gamma\right| & \leq\left|g_{2}\left(\left|x_{k}-\gamma\right|\right)\right| x_{k}-\gamma|<| x_{k}-\gamma \mid,
\end{aligned}
$$

So we have obtained that:

$$
\begin{array}{r}
\left|\hat{e}_{k}\right| \leq g_{1}(r)\left|e_{k}\right| \leq\left(g_{1}(r)\right)^{2}\left|e_{k-1}\right| \leq \cdots \leq\left(g_{1}(r)\right)^{k+1}\left|e_{0}\right|, \\
\left|e_{k+1}\right| \leq g_{2}(r)\left|e_{k}\right| \leq\left(g_{2}(r)\right)^{2}\left|e_{k-1}\right| \leq \cdots \leq\left(g_{2}(r)\right)^{k+1}\left|e_{0}\right|,
\end{array}
$$

Then, we deduce that $\left.y_{k}, x_{k+1} \in\right] \gamma-r, \gamma+r[=I$ and by taking limits in the last expression and using that $\lim _{k \rightarrow+\infty}\left(g_{2}(t)\right)^{k+1}=0$, we get that $\lim _{k \rightarrow+\infty} x_{k}=\gamma$. Then, we have proved a similar result than the one obtained in theorem 1 for the iterative method due to Dong. The uniqueness proof follows in the same way that in Theorem 3.1.

\section{Numerical results}

In this section, first we study the local convergence radius for the third order iterative methods studied in previous sections for some particular examples and by following the theoretical results of this study. For that, we use some equations taken from [3], [7] and [5] to show the comparison of our results with theirs. In each example we give the root, $\gamma$, its multiplicity $m$ and the constants used in (6) and (7).

In table 2 we can see the different values of $r_{i}, i=0,1,2$, described in our theoretical results for Osada's iterative method (5). As it can be observed, the minimum value for the radii is always $r_{2}$. Similar behavior is presented on the two steps method due to Dong, (8), where the sequence of radius is decreasing. Moreover we notice that in this case the value of the local convergence radius is smaller than the one obtained for the one step method due to Osada. This behavior was expected because in this case it is normally used the first step error expression in the second step with the corresponding restriction for finding good bounds. 


\begin{tabular}{|l|c|c|c|c|c|c|}
\hline Exercise & $\gamma$ & $m$ & $k_{1}=k_{m}$ & $k_{2}$ & $k_{0}$ & $p$ \\
\hline \hline$f_{1}(x)=\cos (x)-1$ & 0 & 2 & 1 & 1 & 1 & 1 \\
\hline$f_{2}(x)=\left(x^{5 / 2}-1\right)^{2}$ & 1 & 2 & $\frac{54}{5}-\frac{1}{10} \sqrt{6}$ & $\frac{72}{5}+\frac{1}{30} \sqrt{6}$ & $\frac{72}{5}-\frac{3}{30} \sqrt{2}$ & $\frac{1}{2}$ \\
\hline$f_{3}(x)=x^{2}\left(x^{2}-1\right)$ & 0 & 2 & 12 & 12 & 12 & 1 \\
\hline $\begin{array}{l}f_{4}(x)=\int_{0}^{x} G(x) d x, \\
G(x)=\int_{0}^{x}\left(x+\cos \left(\pi x^{2}\right)\right) d x\end{array}$ & 0 & 2 & $1+2 \pi$ & $2 \pi(1+2 \pi)$ & $2 \pi$ & 1 \\
\hline$f_{5}(x)=\left(\frac{1}{10} x-\frac{1}{15} x^{3 / 2}\right)^{2}$ & $\frac{9}{4}$ & 2 & 2.56 & 0.43 & 6.32 & $\frac{1}{2}$ \\
\hline $\begin{array}{c}f_{6}(x)=x^{5}-8 x^{4}+24 x^{3} \\
-34 x^{2}+23 x-6\end{array}$ & 1 & 3 & 4 & 10 & 10 & 1 \\
\hline
\end{tabular}

Table 1. Nonlinear examples.

\begin{tabular}{||c||c|c|c|c||}
\hline \multicolumn{5}{|c|}{ Osada's method } \\
\hline Examples & $r_{0}$ & $r_{1}$ & $r_{2}$ & $r$ \\
\hline$f_{1}$ & 3.0000 & 0.7418 & 0.6781 & 0.6781 \\
\hline$f_{2}$ & 0.2842 & 0.0733 & 0.0676 & 0.0676 \\
\hline$f_{3}$ & 0.2500 & 0.0646 & 0.0596 & 0.0596 \\
\hline$f_{4}$ & 0.4119 & 0.1025 & 0.0938 & 0.0938 \\
\hline$f_{5}$ & 1.1719 & 0.3033 & 0.2798 & 0.2798 \\
\hline$f_{6}$ & 1.0000 & 0.3822 & 0.3469 & 0.3469 \\
\hline
\end{tabular}

Table 2. Numerical values of local convergence radii for examples given in Tabla (1).

\section{Comparative study}

Now, in order to complete our study we apply the technique described in section 3 to different iterative methods with the aim of performing a numerical comparison by using the different techniques that we mention in the introduction. Specifically, we compare the local convergence radii for the second order modified Newton's method (1) and the third order methods due to Halley (4), whose local convergence study have been performed in [1] and [3] by using the technique that involves divided differences and the assumption conditions given by (2).

First of all, we give the results for obtaining the local convergence radius with the study presented in this paper in the following theorem, whose proof is ommited by it is similar structure with theorem developed in section 3 .

Theorem 5.1. Under the conditions of Lemma 2.2, let $r_{0}=\frac{m+1}{k}$ and $r=\min \left\{r_{i}, i=\right.$ $0,1,2\}$ where $r_{i}$ is the smallest positive root of equation $h_{i}(t)=g_{i}(t)-1, i=1,2$ where functions $g_{i}$ are defined below for different methods. Then, for any initial guess $\left.x_{0} \in\right] \gamma-r, \gamma+r$ [ the sequence $\left\{x_{k}\right\}$ obtained by each iterative method is well defined and verifies that $\left.x_{k+1} \in\right] \gamma-r, \gamma+r[$ for all $k \geq 0$. Moreover the root $\gamma$ is unique in the interval $] \gamma-r_{0}, \gamma+r_{0}[$ and the following error bounds hold for all $k \geq 0$,

$$
\left|x_{k+1}-\gamma\right| \leq\left|g_{2}\left(\left|x_{k}-\gamma\right|\right)\right| x_{k}-\gamma|<| x_{k}-\gamma \mid,
$$

(1) Modified Newton's method, (1), we have

$$
g_{1}(t)=\frac{k t}{m}
$$




\begin{tabular}{||c||c|c|c|c|c||}
\hline \multicolumn{7}{|c|}{ Dong's method } \\
\hline Examples & $r_{0}$ & $r_{1}$ & $r_{2}$ & $r_{3}$ & $r$ \\
\hline$f_{1}$ & 3 & 2.0000 & 0.9252 & 0.2167 & 0.2167 \\
\hline$f_{2}$ & 0.2842 & 0.1895 & 0.0877 & 0.0205 & 0.0205 \\
\hline$f_{3}$ & 0.2500 & 0.1667 & 0.0771 & 0.0181 & 0.0181 \\
\hline$f_{4}$ & 0.4119 & 0.2746 & 0.1270 & 0.0298 & 0.0298 \\
\hline$f_{5}$ & 1.1719 & 0.7813 & 0.3614 & 0.0846 & 0.0846 \\
\hline$f_{6}$ & 1.0000 & 0.7500 & 0.2763 & 0.0230 & 0.0230 \\
\hline
\end{tabular}

Table 3. Numerical values of local convergence radii for examples given in Tabla (1).

and

$$
g_{2}(t)=\frac{k t}{(m+1)(m-k t)}
$$

(2) Halley's method, (4), we get

$$
g_{1}(t)=\frac{k}{m} t+\left(\frac{k^{2}}{2 m^{2}(m+1-k t)}+\frac{p}{m(m+1)(m+2)}\right) t^{2}
$$

and

$$
g_{2}(t)=\frac{\left(\frac{k^{2}}{2 m^{2}(m+1-k t)}+\frac{p}{m(m+1)(m+2)}\right) t^{2}}{1-\left[\frac{k}{m} t+\left(\frac{k^{2}}{2 m^{2}(m+1-k t)}+\frac{p}{m(m+1)(m+2)}\right) t^{2}\right]}
$$

We will use numerical examples given in table 1 where it is shown the values of constants needed in our study $k, p$ and the constant values $K_{m}=k, k_{0}$ and $p$ needed with the technique that uses divided differences and Hölder conditions, (2).

Table 4 shows the radii of local convergence for different iterative methods. We denote with $D D$ when the local convergence study has been performed by using divided differences and denote with $O P$ our proposal that has been established in section 3 . Notice that the computed results are equal for cases where the Hölder's constant $p$ is 1. But second an fourth examples where $p$ is different from 1, there is a small difference. In those examples, our proposal gets a bigger radius, a logical result since our conditions are stricter.

It can be checked that Newton's radius are always bigger than those of the methods of higher order. However, in the case of the methods of order 3 and 4, the difference is less accused. We have to point out the the good values obtained for the radii with Halley's method while Dong's methods has always has reached the smallest radii.

\section{Case of unknown multiplicity}

Having studied the radius of local convergence for the different methods, the goal now is to analyze the behavior of the iterative methods for multiple roots when the value of the multiplicity, $m$ is unknown. In fact, all the iterative methods studied include the value of $m$ in their iterative expression. However, in real problems one can have an 


\begin{tabular}{||c||c|c|c|c|c|c|c||}
\hline \multicolumn{2}{|c|}{ Radius } & \multicolumn{7}{c|}{ Examples } \\
\hline Method & Condition & $f_{1}$ & $f_{2}^{*}$ & $f_{3}$ & $f_{4}$ & $f_{5}^{*}$ & $f_{6}$ \\
\hline \multirow{2}{*}{ M. Newton } & DD & 1.5000 & 0.1421 & 0.1250 & 0.2060 & 0.5859 & 0.6000 \\
\cline { 2 - 8 } & OP & 1.5000 & 0.1421 & 0.1250 & 0.2060 & 0.5859 & 0.6000 \\
\hline \multirow{2}{*}{ Osada } & DD & 0.6781 & 0.0644 & 0.0596 & 0.0938 & 0.2348 & 0.3469 \\
\cline { 2 - 8 } & OP & 0.6781 & 0.0676 & 0.0596 & 0.0938 & 0.2798 & 0.3469 \\
\hline \multirow{2}{*}{ M. Halley } & DD & 1.2679 & 0.1235 & 0.1152 & 0.1762 & 0.4357 & 0.5091 \\
\cline { 2 - 8 } & OP & 1.2679 & 0.1304 & 0.1152 & 0.1762 & 0.5411 & 0.5091 \\
\hline
\end{tabular}

Table 4. Comparing results.

equation with unknown root and unknown multiplicity. How can we proceed in order to apply the method? Our aim in this section is to check if the formulas proposed in [14] for approximating the multiplicity tested in this paper with modified Newton's method are also working with different iterative methods for multiple roots. In this way it is studied if the approximations of the multiplicity allow to give acceptable results with different methods. Finally, some examples are shown to demonstrate their application.

For this section, we use the modified Newton method, Osada's method and an optimum method of fourth order (M4), see [2] defined as

$$
\begin{aligned}
& y_{n}=x_{n}-b \frac{f\left(x_{n}\right)}{f^{\prime}\left(x_{n}\right)}, \\
& x_{n+1}=x_{n}-\left(s_{1}+s_{2} h\left(y_{n}, x_{n}\right)+s_{3} h\left(x_{n}, y_{n}\right)+s_{4} h\left(y_{n}, x_{n}\right)^{2}\right) \frac{f\left(x_{n}\right)}{f^{\prime}\left(x_{n}\right)} .
\end{aligned}
$$

where

$$
\begin{aligned}
h\left(x_{n}, y_{n}\right) & =\frac{f^{\prime}\left(y_{n}\right)}{f^{\prime}\left(x_{n}\right)}, \quad b=\frac{2 m}{2+m}, \quad \mu=1-\frac{b}{m}, \\
s_{1} & =-\frac{1}{4} m\left(-4+2 m+3 m^{2}+m^{3}\right), \\
s_{2} & =\frac{1}{8} m \mu^{m}(2+m)^{3}, \quad s_{3}=\frac{1}{8} m^{4} \mu^{-m}, \quad s_{4} \in \mathbb{R} .
\end{aligned}
$$

Additionally, we used different procedures to estimate the multiplicity $m$ of the root given in [14]:

a) Schröder's method [10]

$$
\begin{aligned}
u_{i} & =\frac{f\left(x_{i}\right)}{f^{\prime}\left(x_{i}\right)}, \\
u_{i}^{\prime} & =\frac{\left(f^{\prime}\left(x_{i}\right)\right)^{2}-f\left(x_{i}\right) f^{\prime \prime}\left(x_{i}\right)}{f^{\prime}\left(x_{i}\right)^{2}}, \\
m_{i} & =\frac{1}{u_{i}^{\prime}} .
\end{aligned}
$$


b) Ostrowsky's method [9]

$$
\begin{aligned}
x_{i+1} & =x_{i}-\frac{f\left(x_{i}\right)}{f^{\prime}\left(x_{i}\right)}, \\
x_{i+2} & =x_{i+1}-\frac{f\left(x_{i+1}\right)}{f^{\prime}\left(x_{i+1}\right)}, \\
m_{i} & =\frac{x_{i}-x_{i+1}}{x_{i}-2 x_{i+1}+x_{i+2}} .
\end{aligned}
$$

c) Traub's method [11]

$$
m_{i}=\lim _{n \rightarrow \infty} \frac{\ln \left(\left|f\left(x_{i}\right)\right|\right)}{\ln \left(\left|\frac{f\left(x_{i}\right)}{f^{\prime}\left(x_{i}\right)}\right|\right)}
$$

d) Straten's method [12]

$$
\begin{aligned}
x_{i+1} & =x_{i}-m_{i} \frac{f\left(x_{i}\right)}{f^{\prime}\left(x_{i}\right)}, \\
m_{i+1} & =\frac{m_{i}}{1-\frac{f\left(x_{i+1}\right) f^{\prime}\left(x_{i}\right)}{f^{\prime}\left(x_{i+1}\right) f\left(x_{i}\right)}} .
\end{aligned}
$$

It is worth noting that the order of approximation of the multiplicity limits the order of the iterative method to which it is applied. Therefore, using the estimations provided by these methods, which are of second order, will limit the order of the resulting iterative method to order 2 . Working with integer multiplicity roots, one can circumvent this difficulty, by rounding the estimation to the nearest integer. In this case, the computational cost can be reduced if one stops estimating the multiplicity after finding two consecutive equal estimations.

These 3 strategies have been applied to equations $f_{2}, f_{5}$ and $f_{6}$ of Section 4 . In the tables, each method is identified by its author abbreviation followed by a digit: 1 if the raw value of the estimated multiplicity is used, 2 if the estimation is rounded, and 3 if one stops estimating the multiplicity when it stabilizes. Additionally, the method is run using the true value of the multiplicity from the beginning, in order to assess the increase of cost due to the multiplicity estimation.

In order to compare the performance of the different strategies, we use the functional evaluations that are used in each iteration. That is, the number of functional evaluations used in the method plus the ones used in the estimation of the multiplicity are taken. Table 5 shows the cost of one iteration on the analyzed method with or without using a multiplicity estimation procedure.redThis aim was partially published in [7].

\begin{tabular}{||c||c|c|c||}
\hline Multiplicity estimation & M. Newton & Osada & M4 \\
\hline known & 2 & 3 & 3 \\
\hline Ostrowski & 4 & 5 & 5 \\
\hline Schröder & 3 & 3 & 3 \\
\hline Traub & 2 & 3 & 3 \\
\hline Straten & 4 & 5 & 5 \\
\hline
\end{tabular}

Table 5. Number of functional evaluations per iteration according to the multiplicity estimation procedure 


\begin{tabular}{|c|c|c|c|c|c|c|c|}
\hline Method & Aprox. & iter & $\rho$ & incr & $f\left(x_{n}\right)$ & mul & $\mathrm{EF}$ \\
\hline \multirow{13}{*}{ M. Newton } & $\mathrm{Ost}_{1}$ & 6 & 2.00 & $7.42 \mathrm{e}-36$ & $2.66 \mathrm{e}-141$ & {$[1.03,1.96,1.99,1.99,1.99,1.99]$} & 24 \\
\hline & $\mathrm{Ost}_{2}$ & 6 & 2.00 & $6.81 \mathrm{e}-26$ & $7.58 \mathrm{e}-101$ & {$[1,2,2,2,2,2]$} & 24 \\
\hline & $\mathrm{Ost}_{3}$ & 6 & 2.00 & $6.81 \mathrm{e}-26$ & $7.58 \mathrm{e}-101$ & {$[1,2,2]$} & 18 \\
\hline & Scho $_{1}$ & 7 & 2.00 & $3.88 \mathrm{e}-22$ & $8.00 \mathrm{e}-86$ & {$[0.52,1.21,1.82,1.99,1.99,1.99,1.99]$} & 21 \\
\hline & $\mathrm{Scho}_{2}$ & 6 & 2.00 & $6.81 \mathrm{e}-26$ & $7.58 \mathrm{e}-101$ & {$[1,2,2,2,2,2]$} & 18 \\
\hline & $\mathrm{Scho}_{3}$ & 6 & 2.00 & $6.81 \mathrm{e}-26$ & $7.58 \mathrm{e}-101$ & {$[1,2,2]$} & 15 \\
\hline & $\operatorname{Trau}_{1}$ & 24 & 1.02 & $6.03 \mathrm{e}-22$ & $2.56 \mathrm{e}-45$ & {$[\ldots, 1.93]$} & 48 \\
\hline & $\operatorname{Trau}_{2}$ & 9 & 2.00 & $4.06 \mathrm{e}-23$ & $9.58 \mathrm{e}-90$ & {$[1,1,1,1,1,2,2,2,2]$} & 18 \\
\hline & $\mathrm{Trau}_{3}$ & 30 & 1.00 & $6.06 \mathrm{e}-11$ & $2.29 \mathrm{e}-20$ & {$[1,1]$} & 60 \\
\hline & Stra $_{1}$ & 5 & 2.42 & $5.58 \mathrm{e}-35$ & $4.80 \mathrm{e}-117$ & {$[1.03,1.96,1.99,1.99,1.99]$} & 20 \\
\hline & $\mathrm{Stra}_{2}$ & 6 & 2.00 & $6.81 \mathrm{e}-26$ & $7.58 \mathrm{e}-101$ & {$[1,2,2,2,2,2]$} & 24 \\
\hline & $\mathrm{Stra}_{3}$ & 5 & 2.00 & $6.54 \mathrm{e}-36$ & $6.45 \mathrm{e}-141$ & {$[1,2,2]$} & 16 \\
\hline & known & 8 & 2.00 & $1.95 \mathrm{e}-38$ & $5.13 \mathrm{e}-151$ & 2 & 16 \\
\hline \multirow{13}{*}{ Osada } & $\mathrm{Ost}_{1}$ & 6 & 2.00 & $6.61 \mathrm{e}-40$ & $3.78 \mathrm{e}-157$ & {$[1.03,1.98,1.99,1.99,1.99,1.99]$} & 30 \\
\hline & $\mathrm{Ost}_{2}$ & 5 & 3.00 & $2.06 \mathrm{e}-35$ & $2.18 \mathrm{e}-207$ & {$[1,2,2,2,2]$} & 25 \\
\hline & $\mathrm{Ost}_{3}$ & 5 & 3.00 & $2.06 \mathrm{e}-35$ & $2.18 \mathrm{e}-207$ & {$[1,2,2]$} & 21 \\
\hline & $\mathrm{Scho}_{1}$ & 7 & 2.00 & $3.88 \mathrm{e}-22$ & $8.01 \mathrm{e}-86$ & {$[0.52,1.21,1.82,1.99,1.99,1.99,1.99]$} & 21 \\
\hline & $\mathrm{Scho}_{2}$ & 5 & 3.00 & $2.06 \mathrm{e}-35$ & $2.18 \mathrm{e}-207$ & {$[1,2,2,2,2]$} & 15 \\
\hline & $\mathrm{Scho}_{3}$ & 5 & 3.00 & $2.06 \mathrm{e}-35$ & $2.18 \mathrm{e}-207$ & {$[1,2,2]$} & 15 \\
\hline & $\operatorname{Trau}_{1}$ & 21 & 1.03 & $7.82 \mathrm{e}-21$ & $1.33 \mathrm{e}-43$ & {$[\ldots, 1.93]$} & 63 \\
\hline & $\operatorname{Trau}_{2}$ & 8 & 3.00 & $1.28 \mathrm{e}-23$ & $1.29 \mathrm{e}-136$ & {$[1,1,1,1,1,2,2,2]$} & 24 \\
\hline & $\operatorname{Trau}_{3}$ & 30 & 1.00 & $6.06 \mathrm{e}-11$ & $2.29 \mathrm{e}-20$ & {$[1,1]$} & 90 \\
\hline & Stra 1 & 5 & 2.43 & $3.13 \mathrm{e}-37$ & $1.88 \mathrm{e}-125$ & {$[1.03,1.96,1.99,1.99,1.99]$} & 25 \\
\hline & $\mathrm{Stra}_{2}$ & 5 & 3.00 & $2.06 \mathrm{e}-35$ & $2.18 \mathrm{e}-207$ & {$[1,2,2,2,2]$} & 25 \\
\hline & $\mathrm{Stra}_{3}$ & 4 & 2.39 & $3.15 \mathrm{e}-36$ & $2.75 \mathrm{e}-212$ & {$[1,2,2]$} & 21 \\
\hline & known & 7 & 2.99 & $4.83 \mathrm{e}-42$ & $3.61 \mathrm{e}-247$ & 2 & 21 \\
\hline \multirow{13}{*}{ M4 } & $\mathrm{Ost}_{1}$ & 6 & 2.00 & $1.92 \mathrm{e}-33$ & $5.57 \mathrm{e}-132$ & {$[1.03,1.93,1.99,1.99,1.99,1.99]$} & 30 \\
\hline & $\mathrm{Ost}_{2}$ & 4 & 4.01 & $1.10 \mathrm{e}-22$ & $1.08 \mathrm{e}-175$ & {$[1,2,2,2]$} & 20 \\
\hline & $\mathrm{Ost}_{3}$ & 4 & 4.01 & $1.10 \mathrm{e}-22$ & $1.07 \mathrm{e}-175$ & {$[1,2,2]$} & 18 \\
\hline & Scho $_{1}$ & 7 & 2.00 & $1.28 \mathrm{e}-36$ & $1.95 \mathrm{e}-144$ & {$[0.52,1.42,1.95,1.99,1.99,1.99,1.99]$} & 21 \\
\hline & $\mathrm{Scho}_{2}$ & 4 & 4.01 & $1.10 \mathrm{e}-22$ & $1.08 \mathrm{e}-175$ & {$[1,2,2,2]$} & 12 \\
\hline & $\mathrm{Scho}_{3}$ & 4 & 4.01 & $1.10 \mathrm{e}-22$ & $1.08 \mathrm{e}-175$ & {$[1,2,2]$} & 12 \\
\hline & $\operatorname{Trau}_{1}$ & 16 & 1.03 & $6.41 \mathrm{e}-22$ & $1.63 \mathrm{e}-46$ & {$[\ldots, 1.93]$} & 48 \\
\hline & $\operatorname{Trau}_{2}$ & 6 & 4.00 & $6.61 \mathrm{e}-41$ & $1.80 \mathrm{e}-321$ & {$[1,1,1,2,2,2]$} & 18 \\
\hline & $\operatorname{Trau}_{3}$ & 30 & 1.00 & $1.08 \mathrm{e}-17$ & $1.13 \mathrm{e}-34$ & {$[1,1]$} & 90 \\
\hline & Stra $_{1}$ & 5 & 2.42 & $2.46 \mathrm{e}-45$ & $6.00 \mathrm{e}-154$ & {$[1.03,1.98,1.99,1.99,1.99]$} & 25 \\
\hline & $\mathrm{Stra}_{2}$ & 4 & 4.01 & $1.10 \mathrm{e}-22$ & $1.08 \mathrm{e}-175$ & {$[1,2,2,2]$} & 20 \\
\hline & $\mathrm{Stra}_{3}$ & 4 & 3.43 & $2.43 \mathrm{e}-76$ & $6.05 \mathrm{e}-605$ & {$[1,2,2]$} & 18 \\
\hline & known & 6 & 3.99 & $7.51 \mathrm{e}-28$ & $4.95 \mathrm{e}-217$ & 2 & 18 \\
\hline
\end{tabular}

Table 6. $f_{2}(x)=\left(x^{5 / 2}-1\right)^{2}, x_{0}=0.5, \gamma=1, m=2$

To be able to make a comparison between the different performances for each method and procedure for multiplicity estimation, the tables 6,7 and 8 show the number of iterations needed to converge, the approximated computational order of convergence (28), the error between the last two iterates, the absolute value of the function evaluated in the last approximation, the multiplicity estimations used in each iteration, and the total cost of the iterations, in terms of the total number of evaluations of the function and its derivatives along the iterations of each method.

$$
\rho=\frac{\log \left(\left|x_{n-1}-x_{n-2}\right| /\left|x_{n}-x_{n-1}\right|\right.}{\log \left(\left|x_{n-2}-x_{n-3}\right| /\left|x_{n-1}-x_{n-2}\right|\right.},
$$

In general, it can be seen that the order of convergence is limited to approximately 2 despite the order of theoretical convergence of the method (rows of tables where the name of the method has subscript 1). It is important that the methods used to estimate the multiplicity give a good approximation.

In the rows where the name of the method has subscripts 2 and 3, the rounding of the approximation of the multiplicity has been made. On most of them, in the cases of sub-index 3 they have a lower cost than sub-index 2 since in case of finding repeated estimates it stops the estimation process and adopts this value as the multiplicity. The Schröder method obtains the best results when estimating the multiplicity, while the Traub method fails to estimate the multiplicity and converge. Also, the cost increment due to the multiplicity estimation is quite moderated. In general, it is less than two times the cost of the same method with known multiplicity. 


\begin{tabular}{|c|c|c|c|c|c|c|c|}
\hline Method & Aprox. & iter & $\rho$ & incr & $f\left(x_{n}\right)$ & $\mathrm{mul}$ & EF \\
\hline \multirow{13}{*}{ M. Newton } & $\mathrm{Ost}_{1}$ & 5 & 2.00 & $8.59 \mathrm{e}-22$ & $3.79 \mathrm{e}-89$ & {$[1.73,1.99,1.99,1.99,1.99]$} & 20 \\
\hline & $\mathrm{Ost}_{2}$ & 6 & 1.99 & $2.80 \mathrm{e}-33$ & $1.71 \mathrm{e}-134$ & {$[2,2,2,2,2,2]$} & 24 \\
\hline & $\mathrm{Ost}_{3}$ & 6 & 1.99 & $2.80 \mathrm{e}-33$ & $1.71 \mathrm{e}-134$ & {$[2,2]$} & 16 \\
\hline & $\mathrm{Scho}_{1}$ & 6 & 2.00 & $5.16 \mathrm{e}-33$ & $1.96 \mathrm{e}-133$ & {$[1.62,1.96,1.99,1.99,1.99,1.99]$} & 18 \\
\hline & $\mathrm{Scho}_{2}$ & 6 & 2.00 & $2.80 \mathrm{e}-33$ & $1.71 \mathrm{e}-134$ & {$[2,2,2,2,2,2]$} & 18 \\
\hline & $\mathrm{Scho}_{3}$ & 6 & 2.00 & $2.80 \mathrm{e}-33$ & $1.71 \mathrm{e}-134$ & {$[2,2]$} & 14 \\
\hline & $\operatorname{Trau}_{1}$ & $\mathrm{nc}$ & - & - & - & - & - \\
\hline & $\operatorname{Trau}_{2}$ & $\mathrm{nc}$ & - & - & - & - & - \\
\hline & $\operatorname{Trau}_{3}$ & $\mathrm{nc}$ & - & - & - & - & - \\
\hline & Stra $_{1}$ & 5 & 2.41 & $1.53 \mathrm{e}-47$ & $7.79 \mathrm{e}-164$ & {$[1.73,1.99,1.99,1.99,1.99]$} & 20 \\
\hline & $\mathrm{Stra}_{2}$ & 6 & 2.00 & $2.80 \mathrm{e}-33$ & $1.71 \mathrm{e}-134$ & {$[2,2,2,2,2,2]$} & 24 \\
\hline & $\mathrm{Stra}_{3}$ & 4 & 2.00 & $8.94 \mathrm{e}-23$ & $1.78 \mathrm{e}-92$ & {$[2,2]$} & 12 \\
\hline & known & 6 & 1.99 & $2.80 \mathrm{e}-33$ & $1.70 \mathrm{e}-134$ & 2 & 12 \\
\hline \multirow{13}{*}{ Osada } & $\mathrm{Ost}_{1}$ & 6 & 2.00 & $8.40 \mathrm{e}-37$ & $7.78 \mathrm{e}-149$ & {$[1.73,1.98,1.99,1.99,1.99,1.99]$} & 30 \\
\hline & $\mathrm{Ost}_{2}$ & 5 & 3.00 & $5.27 \mathrm{e}-53$ & $1.18 \mathrm{e}-317$ & {$[2,2,2,2,2]$} & 25 \\
\hline & $\mathrm{Ost}_{3}$ & 5 & 3.00 & $5.27 \mathrm{e}-53$ & $1.18 \mathrm{e}-317$ & {$[2,2]$} & 19 \\
\hline & Scho $_{1}$ & 6 & 2.00 & $5.16 \mathrm{e}-33$ & $1.96 \mathrm{e}-133$ & {$[1.62,1.96,1.99,1.99,1.99,1.99]$} & 18 \\
\hline & $\mathrm{Scho}_{2}$ & 5 & 3.00 & $5.27 \mathrm{e}-53$ & $1.18 \mathrm{e}-317$ & {$[2,2,2,2,2]$} & 15 \\
\hline & $\mathrm{Scho}_{3}$ & 5 & 3.00 & $5.27 \mathrm{e}-53$ & $1.18 \mathrm{e}-317$ & {$[2,2]$} & 15 \\
\hline & $\operatorname{Trau}_{1}$ & 30 & 1.00 & $7.98 \mathrm{e}-09$ & 1.47 & $\ldots, 0.39]$ & 90 \\
\hline & $\operatorname{Trau}_{2}$ & 30 & 1.32 & 5.74 & 4.2 & {$[\ldots,-1]$} & 90 \\
\hline & $\operatorname{Trau}_{3}$ & 11 & 3.00 & $1.1349 \mathrm{e}-58$ & $1.75 \mathrm{e}-351$ & {$[5,-11,2,2]$} & 33 \\
\hline & Stra $_{1}$ & 4 & 2.41 & $9.40 \mathrm{e}-22$ & $1.59 \mathrm{e}-76$ & {$[1.73,1.99,1.99,1.99]$} & 20 \\
\hline & $\mathrm{Stra}_{2}$ & 5 & 3.00 & $5.27 \mathrm{e}-53$ & $1.18 \mathrm{e}-317$ & {$[2,2,2,2,2]$} & 25 \\
\hline & $\mathrm{Stra}_{3}$ & 3 & 2.40 & $9.36 \mathrm{e}-21$ & $3.70 \mathrm{e}-124$ & {$[2,2]$} & 13 \\
\hline & known & 5 & 3.00 & $5.27 \mathrm{e}-53$ & $1.18 \mathrm{e}-317$ & 2 & 15 \\
\hline \multirow{13}{*}{ M4 } & $\mathrm{Ost}_{1}$ & 5 & 2.00 & $4.09 \mathrm{e}-24$ & $9.04 \mathrm{e}-99$ & {$[1.73,1.99,1.99,1.99,1.99]$} & 25 \\
\hline & $\mathrm{Ost}_{2}$ & 4 & 3.99 & $2.16 \mathrm{e}-55$ & $1.40 \mathrm{e}-442$ & {$[2,2,2,2]$} & 20 \\
\hline & $\mathrm{Ost}_{3}$ & 4 & 3.99 & $2.16 \mathrm{e}-55$ & $1.40 \mathrm{e}-442$ & {$[2,2]$} & 16 \\
\hline & Scho $_{1}$ & 5 & 2.00 & $1.48 \mathrm{e}-21$ & $2.75 \mathrm{e}-88$ & {$[1.61,1.98 .1 .99,1.99,1.99]$} & 15 \\
\hline & $\mathrm{Scho}_{2}$ & 4 & 3.99 & $2.16 \mathrm{e}-55$ & $1.41 \mathrm{e}-442$ & {$[2,2,2,2]$} & 12 \\
\hline & $\mathrm{Scho}_{3}$ & 4 & 3.99 & $2.16 \mathrm{e}-55$ & $1.41 \mathrm{e}-442$ & {$[2,2]$} & 12 \\
\hline & $\operatorname{Trau}_{1}$ & 13 & 1.02 & $3.90 \mathrm{e}-21$ & $2.27 \mathrm{e}-48$ & {$[\ldots, 2.10]$} & 39 \\
\hline & $\operatorname{Trau}_{2}$ & 6 & 10.55 & $9.38 \mathrm{e}-22$ & $1.75 \mathrm{e}-173$ & {$[5,4,3,3,2,2]$} & 18 \\
\hline & $\operatorname{Trau}_{3}$ & 15 & 1.00 & $8.84 \mathrm{e}-21$ & $1.79 \mathrm{e}-46$ & {$[5,4,3,3]$} & 45 \\
\hline & Stra $_{1}$ & 4 & 2.40 & $3.02 \mathrm{e}-25$ & $2.77 \mathrm{e}-89$ & {$[1.73,1.99,1.99,1.99]$} & 20 \\
\hline & $\mathrm{Stra}_{2}$ & 4 & 3.99 & $2.16 \mathrm{e}-55$ & $1.41 \mathrm{e}-442$ & {$[2,2,2,2]$} & 20 \\
\hline & $\mathrm{Stra}_{3}$ & 3 & 3.43 & $7.50 \mathrm{e}-41$ & $1.78 \mathrm{e}-325$ & {$[2,2]$} & 16 \\
\hline & known & 4 & 3.99 & $2.16 \mathrm{e}-55$ & $1.41 \mathrm{e}-442$ & 2 & 8 \\
\hline
\end{tabular}

Table 7. $f_{5}(x)=\left(\frac{1}{10} x-\frac{1}{15} x^{3 / 2}\right)^{2}, x_{0}=2, \gamma=\frac{9}{4}, m=2$

\section{Conclusions}

We obtain the local convergence for several iterative methods without using some sophisticated properties of divided difference and apply the theoretical results to some problems obtaining the local convergence radius, including a comparison with already existing results. Moreover, we perform a study of the behavior of these methods when $m$ is unknown and we use an estimation.

\section{Agreements}

This research was supported by Ministerio de Economía y Competitividad under grant MTM2014-52016-C2-1-2-P and by the project of Generalitat Valenciana Prometeo/2016/089 and SENESCYT of Ecuador (Secretaría de Educación Superior, Ciencia, Tecnología e Innovacin).

\section{Funding}

This work has not funding. 


\begin{tabular}{|c|c|c|c|c|c|c|c|}
\hline Method & Aprox. & iter & $\rho$ & incr & $f\left(x_{n}\right)$ & mul & $\mathrm{EF}$ \\
\hline \multirow{13}{*}{ M. Newton } & $\mathrm{Ost}_{1}$ & 6 & 2.00 & $1.95 \mathrm{e}-39$ & $4.01 \mathrm{e}-234$ & {$[3.43,2.98,2.99,2.99,2.99,2.99]$} & 24 \\
\hline & $\mathrm{Ost}_{2}$ & 6 & 1.99 & $3.95 \mathrm{e}-34$ & $-9.48 \mathrm{e}-202$ & {$[3,3,3,3,3,3]$} & 24 \\
\hline & $\mathrm{Ost}_{3}$ & 6 & 1.99 & $3.95 \mathrm{e}-34$ & $-9.48 \mathrm{e}-202$ & {$[3,3]$} & 16 \\
\hline & Scho $_{1}$ & 6 & 2.00 & $1.57 \mathrm{e}-34$ & $3.76 \mathrm{e}-204$ & {$[3.49,2.95,2.99,2.99,2.99,2.99]$} & 18 \\
\hline & $\mathrm{Scho}_{2}$ & 6 & 2.00 & $3.95 \mathrm{e}-34$ & $-9.48 \mathrm{e}-202$ & {$[3,3,3,3,3,3]$} & 18 \\
\hline & $\mathrm{Scho}_{3}$ & 6 & 2.00 & $3.95 \mathrm{e}-34$ & $-9.48 \mathrm{e}-202$ & {$[3,3]$} & 14 \\
\hline & $\operatorname{Trau}_{1}$ & 21 & 1.02 & $5.10 \mathrm{e}-22$ & $-5.37 \mathrm{e}-69$ & {$\left[\begin{array}{l}\ldots, 2.92] \\
\end{array}\right.$} & 42 \\
\hline & $\operatorname{Trau}_{2}$ & 10 & 2.00 & $3.78 \mathrm{e}-28$ & $-7.33 e-166$ & {$[1,2,2,2,2,2,3,3,3,3]$} & 20 \\
\hline & $\operatorname{Trau}_{3}$ & 30 & 1.00 & $4.85 \mathrm{e}-15$ & $-2.87 \mathrm{e}-44$ & {$[1,2,2]$} & 60 \\
\hline & Stra $_{1}$ & 5 & 2.43 & $4.61 \mathrm{e}-45$ & $4.45 \mathrm{e}-228$ & {$[3.43,2.98,3.00,2.99,3.00]$} & 20 \\
\hline & $\mathrm{Stra}_{2}$ & 6 & 2.00 & $3.95 \mathrm{e}-34$ & $-9.48 \mathrm{e}-202$ & {$[3,3,3,3,3,3]$} & 24 \\
\hline & $\mathrm{Stra}_{3}$ & 5 & 2.00 & $9.88 \mathrm{e}-39$ & $-2.32 \mathrm{e}-229$ & {$[3,3]$} & 14 \\
\hline & known & 6 & 1.99 & $3.95 \mathrm{e}-34$ & $-9.48 \mathrm{e}-202$ & 3 & 12 \\
\hline \multirow{13}{*}{ Osada } & $\mathrm{Ost}_{1}$ & 6 & 2.00 & $8.67 \mathrm{e}-37$ & $6.12 \mathrm{e}-218$ & {$[3.43,2.98,2.99,2.99,2.99,2.99]$} & 30 \\
\hline & $\mathrm{Ost}_{2}$ & 4 & 2.99 & $5.05 \mathrm{e}-23$ & $-2.47 \mathrm{e}-201$ & {$[3,3,3,3]$} & 20 \\
\hline & $\mathrm{Ost}_{3}$ & 4 & 2.99 & $5.05 \mathrm{e}-23$ & $-2.47 \mathrm{e}-201$ & {$[3,3]$} & 16 \\
\hline & Scho $_{1}$ & 6 & 2.00 & $1.57 \mathrm{e}-34$ & $3.76 \mathrm{e}-204$ & {$[3.49,2.95,2.99,2.99,2.99,2.99]$} & 18 \\
\hline & $\mathrm{Scho}_{2}$ & 4 & 2.99 & $5.05 \mathrm{e}-23$ & $-2.47 \mathrm{e}-201$ & {$[3,3,3,3]$} & 12 \\
\hline & $\mathrm{Scho}_{3}$ & 4 & 2.99 & $5.05 \mathrm{e}-23$ & $-2.47 \mathrm{e}-201$ & {$[3,3]$} & 12 \\
\hline & $\operatorname{Trau}_{1}$ & 17 & 1.03 & $4.99 \mathrm{e}-21$ & $-7.86 e-67$ & {$[\ldots, 2.91]$} & 51 \\
\hline & $\operatorname{Trau}_{2}$ & 8 & 2.99 & $8.84 \mathrm{e}-30$ & $-3.83 e-262$ & {$[1,2,2,2,2,3,3,3]$} & 24 \\
\hline & $\operatorname{Trau}_{3}$ & 30 & 1 & $1.71 \mathrm{e}-18$ & $-3.71 \mathrm{e}-55$ & {$[1,2,2]$} & 90 \\
\hline & Stra $_{1}$ & 4 & 2.36 & $9.39 \mathrm{e}-21$ & $8.89 \mathrm{e}-105$ & {$[3.43,2.99,3.00,2.99]$} & 20 \\
\hline & $\mathrm{Stra}_{2}$ & 4 & 2.99 & $5.05 \mathrm{e}-23$ & $-2.47 \mathrm{e}-201$ & {$[3,3,3,3]$} & 20 \\
\hline & $\mathrm{Stra}_{3}$ & 4 & 3.00 & $5.54 \mathrm{e}-55$ & $-5.66 e-489$ & {$[3,3]$} & 16 \\
\hline & known & 4 & 2.99 & $5.05 \mathrm{e}-23$ & $-2.47 \mathrm{e}-201$ & 3 & 12 \\
\hline \multirow{13}{*}{ M4 } & $\mathrm{Ost}_{1}$ & 5 & 2.00 & $9.27 \mathrm{e}-23$ & $1.19 \mathrm{e}-134$ & {$[3.43,2.99,2.99,2.99,2.99]$} & 25 \\
\hline & $\mathrm{Ost}_{2}$ & 4 & 3.99 & $2.67 \mathrm{e}-57$ & $2.63 \mathrm{e}-680$ & {$[3,3,3,3]$} & 20 \\
\hline & $\mathrm{Ost}_{3}$ & 4 & 3.99 & $2.67 \mathrm{e}-57$ & $2.63 \mathrm{e}-680$ & {$[3,3]$} & 16 \\
\hline & Scho $_{1}$ & 5 & 2.00 & $7.97 \mathrm{e}-22$ & $8.34 \mathrm{e}-129$ & {$[3.50,2.97,2.99,2.99,2.99]$} & 15 \\
\hline & $\mathrm{Scho}_{2}$ & 4 & 3.99 & $2.67 \mathrm{e}-57$ & $2.67 \mathrm{e}-57$ & {$[3,3,3,3]$} & 12 \\
\hline & $\mathrm{Scho}_{3}$ & 4 & 3.99 & $2.67 \mathrm{e}-57$ & $2.67 \mathrm{e}-57$ & {$[3,3]$} & 12 \\
\hline & $\operatorname{Trau}_{1}$ & 14 & 1.03 & $5.08 \mathrm{e}-21$ & $-1.09 \mathrm{e}-67$ & {$[\ldots, 2.91]$} & 42 \\
\hline & $\operatorname{Trau}_{2}$ & 7 & 3.99 & $1.56 \mathrm{e}-57$ & $-4.27 \mathrm{e}-683$ & {$[1,2,2,2,3,3,3]$} & 42 \\
\hline & $\operatorname{Trau}_{3}$ & 25 & 1 & $7.92 \mathrm{e}-21$ & $-5.22 \mathrm{e}-63$ & {$[1,2,2]$} & 75 \\
\hline & Stra $_{1}$ & 4 & 2.35 & $4.15 \mathrm{e}-24$ & $-4.88 \mathrm{e}-123$ & {$[3.43,2.99,3.00,2.99]$} & 20 \\
\hline & $\mathrm{Stra}_{2}$ & 4 & 3.99 & $2.67 \mathrm{e}-57$ & $-2.62 \mathrm{e}-680$ & {$[3,3,3,3]$} & 20 \\
\hline & $\mathrm{Stra}_{3}$ & 3 & 3.39 & $3.98 \mathrm{e}-34$ & $-3.19 \mathrm{e}-402$ & {$[3,3]$} & 16 \\
\hline & known & 4 & 3.99 & $2.67 \mathrm{e}-57$ & $-2.62 \mathrm{e}-680$ & 3 & 12 \\
\hline
\end{tabular}

Table 8. $f_{6}(x)=x^{5}-8 x^{4}+24 x^{3}-34 x^{2}+23 x-6, x_{0}=0.8, \gamma=1, m=3$

\section{References}

[1] I. Argyros, On the convergence and application of Newtons method under weak Hölder continuity assumptions, International Journal of Computer Mathematics. 80 (2003), pp. 767-780.

[2] Jose L. Hueso, E. Martínez, C. Teruel, Determination of multiple roots of nonlinear equations and applications, Journal of Mathematical Chemistry. 53 (2015), pp. 880-892.

[3] W. Bi, H. Ren, Q. Wu, Convergence of the modified Halleys method for multiple zeros under Hölder continuous derivative, Numerical Algorithms. 58 (2011), pp. 497-512.

[4] X. Zhou, X. Chen, Y. Song, On the convergence radius of the modified Newton method for multiple roots under the center Hölder condition, Numerical Algorithms, 65 (2014), pp. 221-232.

[5] X. Zhou, Y. Song, Convergence radius of Osada's method under center-Hölder continuous condition, Applied Mathematics and Computation. 243 (2014), pp. 809-816.

[6] C. Dong, A basic theorem of constructing an iterative formula of the higher order for computing multiple roots of an equation, Mathematica Numerica Sinica. 11 (1982), pp. 445-450.

[7] D. Alarcón, F. Cevallos, J.L. Hueso, and E. Martínez, A Comparative Study of the Local Convergence Radius of Iterative Methods for Multiple Roots, Modelling for Engineering and Human Behavior. (2017)

[8] Apostol Tom M, One-Variable Calculus, with an introduction to Lineal Algebra. Blaisdell Publishing Company, Waltham, Massachusetts (1967).

[9] J. Ortega, Solution of Equations in Euclidean and Banach Spaces, SIAM Review. 16 (1974), pp. 564-564.

[10] E. Schröder, Ueber unendlich viele Algorithmen zur Auflösung der Gleichungen, Mathematische Annalen. 16 (1870), pp. 317-365.

[11] J.K. Traub, Iterative methods for the solution of equations, Mathematische Annalen. 
(1964), pp. 317-365.

[12] M. Vander Straten, H. Van de Vel, Multiple root-finding methods, Journal of Computational and Applied Mathematics. 40 (1992), pp. 105-114.

[13] Osada, N. An optimal multiple root-finding method of order three. Journal of Computational and Applied Mathematics 51, 1 (1994), pp. 131-133.

[14] J.M. McNamee, A comparison of methods for accelerating convergence of Newton's method for multiple polynomial roots, ACM SIGNUM Newsletter. 33 (1998), pp. 17-22. 